\title{
Richard, Are We There Yet? An Internet of Nano Things Information System Architecture
}

\author{
Gero Strobel \\ University Duisburg-Essen \\ gero.strobel@uni-due.de
}

\begin{abstract}
Richard Feynman's visionary lecture at Caltech, which dates back more than half a century, is regarded as the birth of today's nanotechnology. Over time, many nanotechnology-based approaches, methods, and innovations have been adopted in almost all research disciplines. This nano-centered evolution is currently transforming the traditional paradigm of the Internet of Things into the Internet of Nano Things. However, the focus here is not on the mere adaptation of down scaling and is instead primarily on the effects and characteristics that result from this new scale. To fully exploit these new possibilities and combine them with existing added-value technologies, it is not sufficient to miniaturize existing Internet-of-Thingsbased systems-they must be developed from scratch. Against this background, this study examines the main areas of nanotechnology in combination with the Internet of Things phenomenon and, based on an exploratory literature search, presents an information system architecture for IoNT-based information systems.
\end{abstract}

\section{Introduction}

In 1959, Richard Feynman delivered his visionary lecture "There's Plenty of Room at the Bottom" at the American Physical Society Symposium at Caltech, and this lecture would later go down in history as the birth of nanotechnology. During his lecture, Feynman did not even mention the term nanotechnology, rather he spoke about the possibilities of manipulating matter at the atomic level and his vision [19]:.

"What I have demonstrated is that there is room.."

"I now want to show that there is plenty of room.."

Almost 15 years after his lecture, Feynman's vision became a reality when Norio Taniguchi first used and published the term nanotechnology in the context of his semiconductor research. Nanotechnology experienced a complete renaissance

\author{
Judith Mittnacht \\ University Duisburg-Essen \\ judith.mittnacht@uni-due.de
}

beyond the classical research disciplines at the end of the 20th century with the passing of the 21st Century Nanotechnology Research and Development Act [23]. Based on this presidential decree of George W. Bush, the USA made nanotechnology a national matter, which not only brought the topic into the media spotlight but also provided appropriate financial resources for research and development [23]. With this development, the nanotechnological approach has penetrated many research areas and domains over the years.

One such domain is the Internet of Things (IoT), which is described as "a worldwide network of interconnected objects uniquely addressable, based on standard communication protocols," and based on smart objects equipped with sensors and actuators. Through the integration of nanotechnological approaches, primarily the scaling of smart objects and sensors in a network, the paradigm shifted around the prefix "nano," leading to a further paradigm shift toward the Internet of Nano Things (IoNT).

Within the literature, various publications address the complex subject of the IoNT, including a variety of system designs and architectural approaches. However, most of these approaches focus on individual components (bio-node) or dedicated and often strongly nano-oriented aspects, such as the communication of individual nodes or their energy supply. However, to develop a holistic information system, it is not sufficient to consider individual system components in isolation from the system's context.

How must an information system be designed from an architectural perspective to satisfy the demands of IoNT-based systems?

Against this background, the objective of this study was to develop an information system architecture for IoNT-based systems. The approach used for this study was a systematic literature search. 
The remainder of this paper is structured as follows. It begins with a theoretical consideration of the central subjects related to nanotechnology and the IoNT. We then present a detailed examination of the research process. Based on this, the developed information system architecture, as well as its components and views, are described in depth. The paper concludes with commentaries on the limitations of the work and suggestions for future research.

\section{Theoretical Background}

\subsection{Nanotechnology and Nanoscience}

The term nanotechnology has now been in scientific usage since the mid-1970s, but there is still no generally accepted definition of the term and the associated research discipline. Thus, the term instead represents a combination of different research disciplines, such as materials science, physics, and chemistry and their methodological approaches, processes, and findings [7, 44].

The most widely used definition is that of the National Nanotechnology Initiative, which defines nanotechnology as "the understanding and control of matter at dimensions of roughly 1-100 nanometers, where unique phenomena enable novel applications. Encompassing nanoscale science, engineering and technology, nanotechnology involves imaging, measuring, modeling, and manipulating matter at this length scale" [32].

On a more abstract level and following Richard Feynman's vision, nanotechnology can be described as the development and utilization of structures with a scale between one molecule and 10 nanometers, whereby the material properties of the starting material change on the basis of the size adjustment. This makes it possible to construct and manipulate objects and structures on a molecular or atomic level [39].

\subsection{Internet of Nano Things}

The Internet of Things (IoT) in its current form is an integral part of our everyday private and professional lives. McKinsey predicts that by the year 2023, more than 43 billion smart devices will be in use in a variety of domains (e.g., smart homes, smart health) [15].

Nanofication, which is the transfer of these smart objects to the nanoscale and the associated paradigm shift towards the Internet of Nano Things, will lead to not only new applications in existing domains [21, 42] but also completely new application scenarios that have not yet been implemented [3]. A pioneer in this field is the medical sector, as regions that were previously difficult or impossible to access are now becoming accessible because of the size and associated characteristics of the newly developed objects [2, 3]. Nano-nodes, the counterpart of smart objects in traditional IoT systems, can be used to treat cancer cells at the cellular level or to administer drugs with pinpoint accuracy $[3,5]$.

Bio-nano-nodes and the Internet of Bio-Nano Things (IoBNT) are the counterparts of artificially produced nano-machines [3]. These are obtained by synthesizing biological cells that contain nanotechnology and function primarily in biological environments [3].

\section{Research Method}

As the research field of the Internet of Nano Things is rather new and constantly evolving, the approach used for developing an architecture was a systematic literature search, according to Webster and Watson [47] as well as Vom Brocke [46].

The aims of the literature search were to identify the widest possible range of different system architectural-relevant objects, characteristics, and entities and, with this information, gain a fundamental understanding of the domain and its technological and systemic approach. To cover a broad spectrum of various approaches and architectures, the literature search was not limited to the central domain term "Internet of Nano Things" but was extended by its biological counterpart "Internet of Bio-Nano Things" and its acronyms.

Against this background, the search string "("Internet of Nano Things" OR "Internet of Bio-Nano Things" OR "IoNT" OR "IoBNT")" was applied in six major databases of the field (e.g., AISeL, IEEE; ACM, etc.) to cover a wide range of literature with diverse focuses. To guarantee an appropriate level of quality, other quality factors were defined in addition to the search string itself. Thus, only publications published in German or English under peer review were considered in the search. The search period was not limited and extended from 1966 to 2020.

Based on the search string and quality factors, 380 publications were initially identified within the databases. Based on the abstracts and keywords, 75 of these were independently deemed relevant. After filtering the duplicates, the remaining 55 papers were subjected to an independent full-text analysis, during which 21 architectures were identified. To ensure the identification of as many different architectural approaches as possible, a forward and backward search was carried out based on the publications whose architectural approaches were 
determined to be relevant. As a result, three additional approaches were identified, increasing the total number to 24 . Special care was taken to include only independent approaches and to exclude referenced approaches or to use the corresponding primary source.

Table 1. Architectural components

\begin{tabular}{|c|c|c|c|c|c|c|}
\hline \multicolumn{3}{|c|}{ Reference } & \multicolumn{4}{|c|}{ Architectural Component } \\
\hline$[34]$ & End User & $\begin{array}{c}\text { Server / } \\
\text { Cloud }\end{array}$ & Smartphone & $\begin{array}{c}\text { Bio-Cyber } \\
\text { Interface }\end{array}$ & $\begin{array}{c}\text { Nano } \\
\text { Sensor }\end{array}$ & $\begin{array}{l}\text { Human } \\
\text { Organs }\end{array}$ \\
\hline [2] & \multicolumn{2}{|c|}{ Healthcare Provider } & Gateway & $\begin{array}{c}\text { Nano-Micro } \\
\text { Interface } \\
\text { Device }\end{array}$ & $\begin{array}{l}\text { Nano } \\
\text { Router }\end{array}$ & $\begin{array}{l}\text { Nano } \\
\text { Node }\end{array}$ \\
\hline [10] & \multicolumn{2}{|c|}{ Nano-Networks (EM/ MC) } & Microgateway & $\begin{array}{c}\text { Context } \\
\text { Managment } \\
\text { Layer }\end{array}$ & $\begin{array}{l}\text { Service } \\
\text { Layer }\end{array}$ & $\begin{array}{l}\text { Nano/ } \\
\text { Micro } \\
\text { Sensors }\end{array}$ \\
\hline [12] & \multicolumn{2}{|c|}{ Medical Personnel } & Access Point & $\begin{array}{l}\text { Bio-Cyber } \\
\text { Interface }\end{array}$ & $\begin{array}{c}\text { Nano } \\
\text { Devices }\end{array}$ & $\begin{array}{l}\text { Nano } \\
\text { Sensors }\end{array}$ \\
\hline [29] & Operator & $\begin{array}{l}\text { Computation } \\
\text { Center }\end{array}$ & Gateway & $\begin{array}{l}\text { Integrated } \\
\text { Computation } \\
\text { Center }\end{array}$ & \multicolumn{2}{|c|}{ Sensor/Actuator } \\
\hline [13] & $\begin{array}{l}\text { Healthcare } \\
\text { Provider }\end{array}$ & $\begin{array}{c}\text { Mini } \\
\text { Transceiver }\end{array}$ & $\begin{array}{l}\text { Bio-Cyber } \\
\text { Interface }\end{array}$ & Nano Devices & \multicolumn{2}{|c|}{ Nanoparticles } \\
\hline [16] & $\begin{array}{c}\text { Analysis and } \\
\text { Control Station } \\
\end{array}$ & Gateway & $\begin{array}{c}\text { Body } \\
\text { Area Network }\end{array}$ & \begin{tabular}{|c|} 
Inplantable \\
Device
\end{tabular} & \multicolumn{2}{|c|}{ In-Body Network } \\
\hline [18] & \begin{tabular}{c|l}
$\begin{array}{c}\text { Medical } \\
\text { Personal }\end{array}$ & I \\
\end{tabular} & Nano Receiver & $\begin{array}{c}\text { Access } \\
\text { Point } \\
\end{array}$ & $\begin{array}{c}\text { Encrypted Bio- } \\
\text { Cyber Device } \\
\end{array}$ & \multicolumn{2}{|c|}{ Nano Sensor } \\
\hline [28] & \multicolumn{2}{|c|}{$\begin{array}{c}\text { Healthcare } \\
\text { Provider / Server }\end{array}$} & $\begin{array}{c}\text { Personal } \\
\text { Device }\end{array}$ & $\begin{array}{c}\begin{array}{c}\text { Body Area } \\
\text { Network }\end{array} \\
\end{array}$ & \multicolumn{2}{|c|}{ Sensor } \\
\hline [4] & Smart Device & Gateway & Nano Router & $\begin{array}{c}\text { Nano Cluster } \\
\text { Head }\end{array}$ & \multicolumn{2}{|c|}{ Nanomachine } \\
\hline [36] & Healthcare Server & $\begin{array}{l}\text { Monitoring } \\
\text { Devices }\end{array}$ & Nano Interface & Nano Router & \multicolumn{2}{|c|}{ Nano Node } \\
\hline [37] & $\begin{array}{c}\text { Healthcare } \\
\text { Provider / } \\
\text { User }\end{array}$ & Gateway & Microgateway & $\begin{array}{l}\text { Nano-Micro- } \\
\text { Interface }\end{array}$ & $\begin{array}{l}\text { Nano } \\
\text { Router }\end{array}$ & $\begin{array}{l}\text { Nano } \\
\text { Sensor }\end{array}$ \\
\hline [3] & \multicolumn{2}{|c|}{ Bio-Cyber Interface } & \multicolumn{2}{|c|}{ Nano Sensor } & \multicolumn{2}{|c|}{ Artificial Cell } \\
\hline [5] & Gateway & $\begin{array}{c}\text { Nano Micro } \\
\text { Interface }\end{array}$ & \multicolumn{2}{|c|}{ Nano Router } & \multicolumn{2}{|c|}{ Nano Node } \\
\hline [27] & Micro Link & Nano Link & Gateway & \begin{tabular}{|c|}
$\begin{array}{c}\text { Nano Micro } \\
\text { Interface }\end{array}$ \\
\end{tabular} & Nano Router & $\begin{array}{l}\text { Nano } \\
\text { Node } \\
\end{array}$ \\
\hline [33] & Doctor & Cloud & \multicolumn{2}{|c|}{ Bio-Cyber Interface } & \multicolumn{2}{|c|}{ Nano Device } \\
\hline [9] & $\begin{array}{l}\text { Physician } \\
\text { Monitoring }\end{array}$ & Wireless Link & \multicolumn{2}{|c|}{ Bio-Cyber Interface } & \multicolumn{2}{|c|}{$\begin{array}{l}\text { Molecular } \\
\text { Communication } \\
\text { Network }\end{array}$} \\
\hline [31] & App Provider & Gateway & Nano Router & Nano Sensor & $\begin{array}{c}\text { Nano-Micro } \\
\text { Interface }\end{array}$ & \begin{tabular}{|c|} 
Nano/ \\
Micro \\
Link \\
\end{tabular} \\
\hline [41] & Smart Device & Nano Router & \multicolumn{2}{|c|}{ Nano Controller } & \multicolumn{2}{|c|}{ Nano Device } \\
\hline [17] & $\begin{array}{c}\text { Medical } \\
\text { Professional } \\
\end{array}$ & Access Point & Bio-Cyber Device & Nano Receiver & \multicolumn{2}{|c|}{ Nano Sensor } \\
\hline [20] & \multicolumn{2}{|c|}{ Medical Personnel } & Access Point & $\begin{array}{l}\text { Bio-Cyber } \\
\text { Interface }\end{array}$ & \multicolumn{2}{|c|}{ Nano Device } \\
\hline [38] & \multicolumn{2}{|c|}{ Application Layer } & \multicolumn{2}{|c|}{ Network Layer } & \multicolumn{2}{|c|}{ Nano Things Layer } \\
\hline [43] & In-Body Network & Gateway & \multicolumn{2}{|c|}{ Analysis and Control Station } & \multicolumn{2}{|c|}{ Nano Communication } \\
\hline [14] & In-Body Network & $\begin{array}{c}\text { Medical } \\
\text { Presonnel }\end{array}$ & \multicolumn{2}{|c|}{ Bio-Cyber Interface } & Nano Ser & ensor \\
\hline
\end{tabular}

The architecture approaches and components identified within the result set differ greatly in terms of naming and descriptions, as well as the structure of the architecture itself. To present a clear overview of the results, the entities were organized by size, starting with the largest objects (Table 1).

Strikingly, most of the architectures originate from a medical- or healthcare-oriented application scenario. Furthermore, the user appears to play a special role in the system context, especially in healthcare-oriented application scenarios, because almost all users were represented as separate entities and even precisely described or differentiated based on their activity (e.g., patient, healthcare professional).

Regardless of the design of the individual components and the presented application scenario, the architectures are all essentially based on the same basic components.

Following the methodological approach of the concept matrix (Table 2), according to Webster and Watson [47], two central architecture views, the macro and nano perspectives, and seven essential (nanonode, nano-router, smart service etc.) architecture components were identified from the results of the literature search following a detailed examination and the aggregation of the extracted individual components.

Table 2. Concept matrix

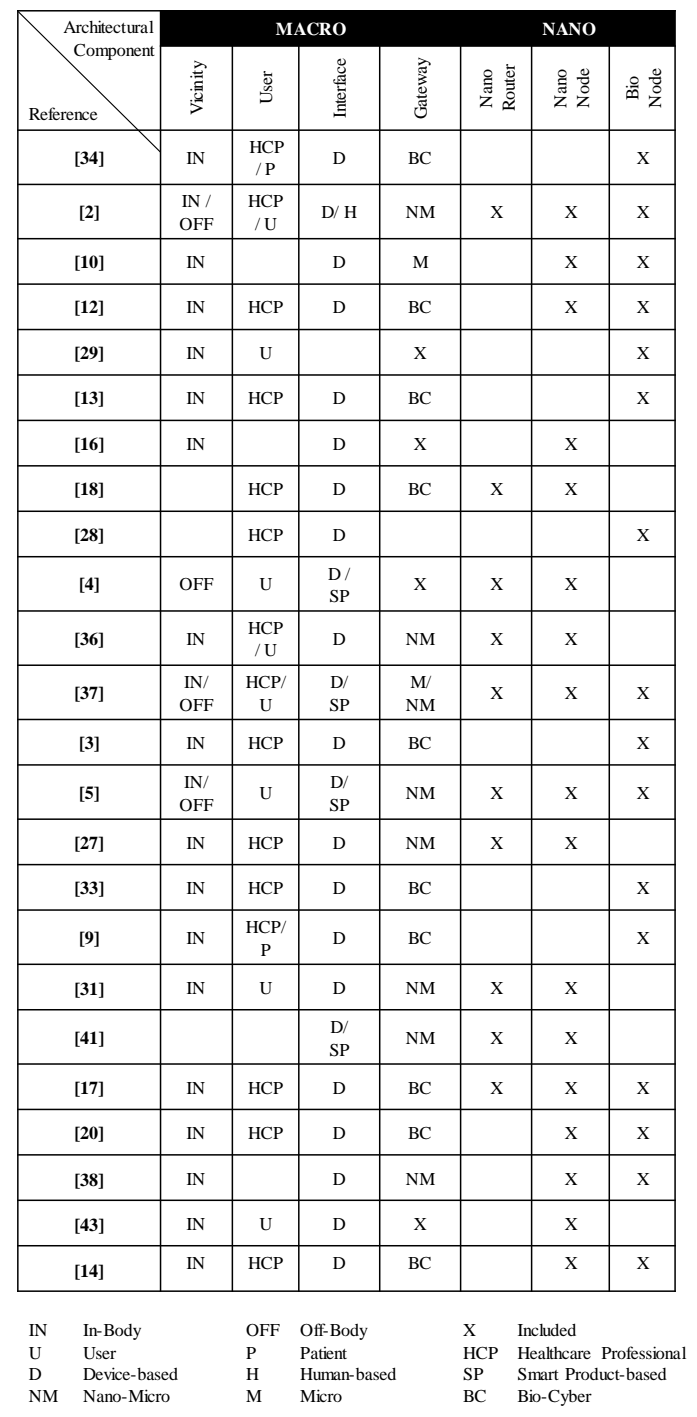

\section{Internet of Nano Things Architecture}

Similar to existing information system architecture concepts (e.g., ARIS [40]), the developed architecture approach (Figure 1) follows a view- 


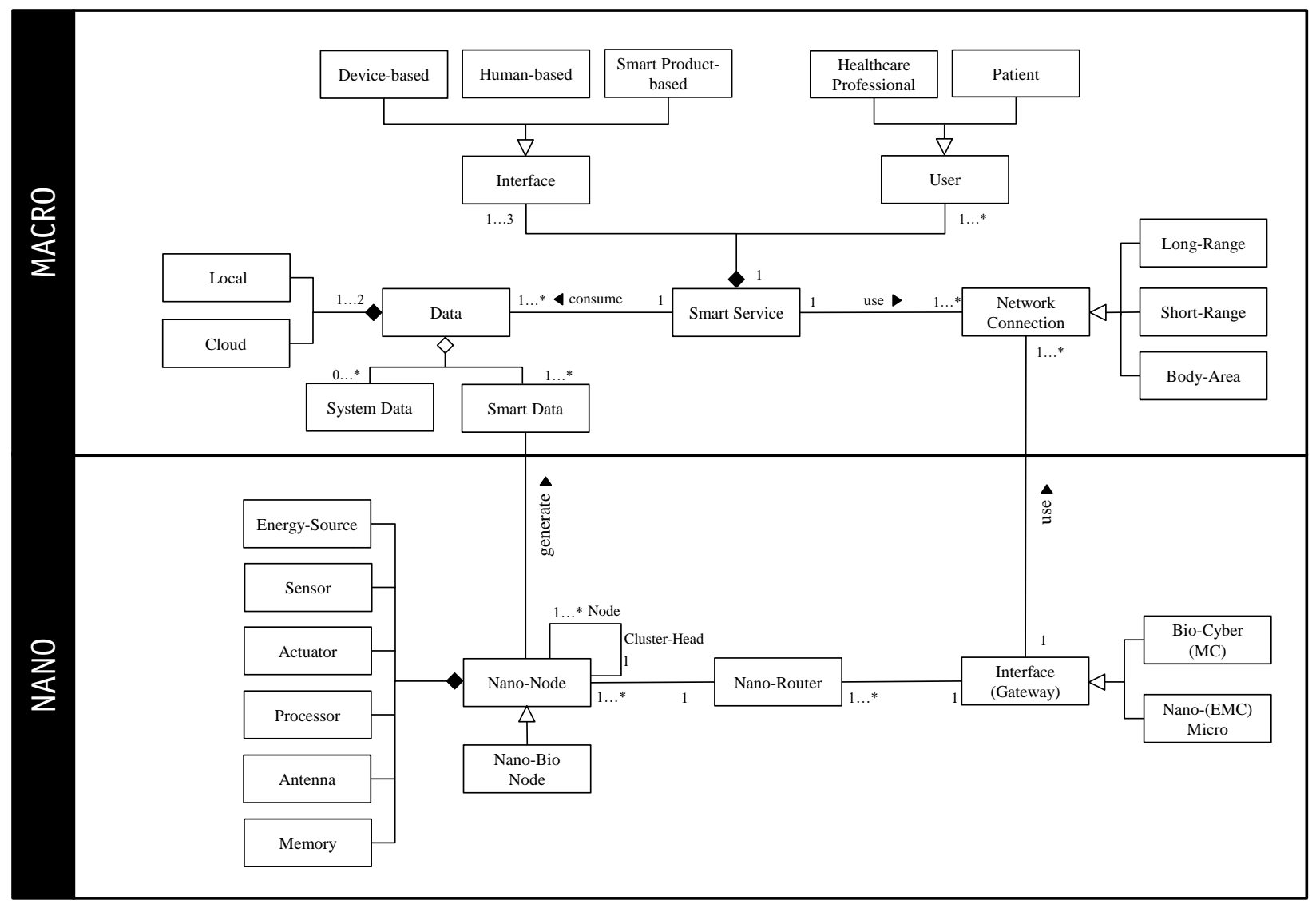

Figure 1. IoNT system architecture

oriented concept. A distinction is made between the macro and nano perspectives. Within the architecture, the macro-view represents system entities and processes that are comprehensible to the naked eye, whereas the nano-view includes the nano-focused system entities and their interaction.

\subsection{Macro-View}

The central system interface and points of interaction between the system and user are intelligent services, so-called Smart Services. Depending on the user group and application, these services provide the user with a dedicated and individualized range of functions, which is a key difference from traditional IoT-based systems (e.g., Smart Home, Smart Fitness, etc.). These services are strongly end-user-oriented because of the complexity of the system and the fields of application. In particular, however, this becomes visible in the health sector, where the nano-machines and thus the entities monitored or controlled by the system are not located in the surrounding environment, such as an intelligent lamp in the context of a Smart Home; in most applications, these are placed in the human body. Regardless of the user group and use case, the smart services and the information provided by them can be consumed by users in three different ways [35]. In most cases, a device-oriented approach is used involving browserenabled devices, such as smartphones, tablets, or even notebooks. It is also possible for the user to interact with the system via smart products (smart productoriented), such as intelligent fitness watches or blood glucose meters. Despite the possibilities currently offered by digital services, personal exchange is essential, especially in critical and highly personal domains such as medicine (human-oriented) [35]. The main interfaces make bidirectional interaction possible, not only allowing the user to consume information but also enriching the system with additional information.

As with traditional IoT-based systems, the system users differentiated here basically depend on the domain and the dedicated use case. Within the architecture, the user is represented as a generic entity, which allows the

architecture user to define their individual characteristics. Based on the user types extracted during the literature analysis, the generic entity was extended to the user group from the healthcare sector, 
which included patient and healthcare professional (e.g. surgeon, nurse).

The information and data on which they are based is another essential component of IoNT-based information systems and thus of the architectural approach. In the context of IoNT-based information systems, data can be divided into three basic types: intelligent, systemic, and external data. Smart data are the data collected by nano-nodes and their sensors about their environment, their operating state, or their interaction with other system entities. In addition to smart data, the system also has other system and operationally relevant data, such as master or login data, which are referred to as system data. It is also possible to enrich the data available in the system with external data via appropriate interfaces to, for example, obtain higher-quality results for decision support or analyses. As with classic information systems, the data can be stored both locally and in the cloud. In contrast to independent data storage, cloud storage reduces management overhead and allows non-domain experts in particular to focus on the primary application scenario [48].

In contrast to conventional IoT-based information systems, the network component in IoNT-based systems not only connects the individual system entities and enables their communication but also functions as a link between the different views. It is important to differentiate between different communication types based on the view. Within the macro-view, the network topologies can be divided into long-range, short-range, and body area networks [8], the last of which is a special form of short-range communication using in body-based systems [6].

Short-range communication can thus be classified as transmission within a radius of a few centimeters up to approximately 100 meters using protocols such as Wi-Fi, Bluetooth, and ZigBee [30, 45]. A specialized form of this communication protocol is the so-called body area network. This type of wireless network connects devices that are either worn very close to the body, usually directly on the skin, or permanently implanted [24]. If, on the other hand, transmissions over several kilometers are necessary for system success, long-range technologies based on existing infrastructure components, such as $5 \mathrm{G}$ or low-power wide-area networks (LPWAN), are used [11]. For most future use cases and applications, however, a combination of both technologies, as is currently the case with non-nanofocused IoT systems, will certainly be advantageous.

\subsection{Nano-View}

The nano-view includes all nanofocused system entities and describes their architectural interaction. The fields of application of nano-components can be divided into three areas: in-body, on-body, and offbody. A combination of several fields of application within one system is possible [3-5, 41]. In the case of in-body or intra-body networks, the field of application of nano-nodes is the human [3, 4] or animal [5] body. For this purpose, the nano-machines are either ingested in the form of a pill or injected through a cannula. The primary field of application is the medical sector [16, 37]. The primary area of application of on-body networks is also living organisms. However, the sensors are positioned directly on the body or on the body surface and not inside the body, either as part of a body area network or in the form of a portable device (e.g., a smart watch). On the other hand, the area of application of off-body networks is the personal environment or the environment [2]. Here, the field of application is only limited by the nature and properties of the nano-nodes. For example, they can be integrated into the home, car, streets, or buildings $[2,26]$. The central areas of application for off-body networks are smart cities in the context of comprehensive and continuous air and building monitoring [22].

The basic elements of the nano-layer are nanonodes with a size between 100 nanometers and at most one micrometer [2, 43]. They represent the nanofocused counterpart to smart products in conventional IoT systems. In contrast to their IoT equivalent, however, they have only rudimentary computing power, highly limited storage capacities, low energy storage capacities, and limited communication possibilities, which means that they can only transfer data over short distances $[2,37]$. The nano-node itself is in turn composed of individual nano-components (e.g., sensor, antenna, processor, etc.).

The individual components perform functions comparable to those of conventional smart products. For example, the antenna serves as a communication medium by receiving, decoding, and transmitting messages via differentiated wave types. The processors form the processing and control units that make it possible for the node to perform tasks. Both artificial and biological nano-batteries are used as energy storage devices to provide the energy supply. In contrast, the integrated storage medium, based on its storage volume, only serves as a buffer until the collected data can be passed on $[1,25]$.

The central components of the nano-nodes are the sensors and actuators. These can be used to measure 
various physical (e.g., electromagnetic fields, light, heat, $\mathrm{pH}$ value, etc.) and physiological (e.g., blood pressure, heartbeat, insulin concentration, etc.) parameters. It is also possible to equip the nodes with an optical sensor and then take pictures of areas that are difficult to access or of dedicated regions of the body [14, 28, 29, 37]. Bio-nano-nodes represent the biological counterpart to artificially constructed nanonodes and imitate biological cells or enzymes in both structure and communication behavior [37].

However, depending on the intended use and application area, an individual node is often not sufficient, and it is necessary to use a cluster of several hundred or even several thousand nodes $[5,12]$. Each of these clusters has a cluster head, which is responsible for collecting and forwarding the data. In the context of nano-clusters, nano-nodes can act as a simple node or as a cluster head [5, 33, 41].

Because of their limited resources, when nanonodes need to transfer the acquired data to entities outside the nano-network, the data must first be transferred to a nano-router [4, 29, 37]. Nano-routers have a higher computing power than nano-nodes. This gives them the ability to both aggregate the information collected by the nano-nodes and control the behavior of the nodes by sending binary commands such as "on" and "off." The nano-routers also have access to the routing and path information of the network, which enables them to target dedicated areas. However, these capabilities also affect the size of the nano-router, making them much larger than nano-nodes, which in turn makes positioning more difficult, particularly in in-body scenarios [2, 37]. The primary task of the nano-router is and remains establishing a connection with the central interface (gateway) and transmitting the received data to it [2, 4].

The interface in turn aggregates the information from the individual nano-routers and is responsible for transmitting this information via a network connection to the respective receiving devices outside the nanonetwork [14]. The interface can be a nano-microinterface or a bio-cyber-interface, depending on the type of communication. In the case of electromagnetic wave communication, a nano-micro interface is used, which enables direct, bidirectional communication between the nano and macro ranges [2, 10, 37]. Molecular communication, on the other hand, requires the use of a bio-cyber interface, which converts the collected biochemical information into electromagnetic communication and makes it readable for macro-view entities [3, 14].

\section{Conclusion and Outlook}

The and the smart products associated with it are now present in almost all areas of private and professional life. By 2023, more than 43 billion smart objects are expected to be in use in a wide variety of domains, including system-critical ones such as healthcare (Smart Health) or energy supply (Smart Grid) [15].

Based on the technological developments in the next few years and the associated possibility of using smart objects on a large scale even in the nanofocused state, this trend becomes more likely. In contrast to research in traditional IoT areas, IoNT-based research is only just beginning.

Against this background, this paper presents a systematic and methodologically sound framework for the analysis and development of IoNT-based information systems based on a layer-oriented information system architecture. This framework can be used as an analysis tool for existing systems to discover white spots within an existing architecture as well as a blueprint for developing new systems. The architecture was developed to be both domain and use case neutral, making it suitable for a wide range of scenarios. Based on the experience gained from the literature search, we see the medical sector as the primary application sector for the technology. The developed architecture was based on a systematic literature analysis of over 350 publications and the resulting aggregation of 24 architectural approaches available in the literature.

Despite the relevant theoretical and practical implications of this study, it has some limitations. First, as a general limitation, although the architecture presented here is scientifically sound, it is still only a theoretical model and has been proposed as a prototype, with the intention of subjecting it to further research. In addition, this architecture based on the literature is limited to two relatively specialized user groups, which should be extended in future studies.

Another limitation relates to a central aspect of literature analyses: the selection of the studies that later formed the basis of the developed architectural. Despite the use of quality criteria and inclusion and exclusion criteria, this selection is always influenced to a certain extent by the subjectivity of the researcher. Thus, to ensure the highest possible degree of objectivity in the selection and aggregation processes (Table 2), they were performed by two researchers independently, and the results were synchronized in a subsequent step. In cases where it was not clear whether publications should be removed from the selection based on quality factors or exclusion criteria, the decision was discussed in a collegial consultation. 
The same process was used for the aggregation and conceptualization steps.

The presented view-oriented information system architecture provides researcher with a methodologically sound foundation for future research and offers room for expansion. A central focus should be placed on the nano-view and the associated architectural components when further developing the architecture. Additional aspects to be considered should be based on current issues within the IoNT field, such as the key aspects of security and computing power.

Finally, this developed architecture provides the first holistic insights into the development of Internet of Nano Things-based information systems and creates a conceptual basis for future research within the field.

\section{References}

[1] Akyildiz, I.F., F. Brunetti, and C. Blázquez, "Nanonetworks: A new communication paradigm", Computer Networks, 52(12), 2008, pp. 2260-2279.

[2] Akyildiz, I.F. and J.M. Jornet, "The Internet of nanothings", IEEE Wireless Communications, 17(6), 2010, pp. 58-63.

[3] Akyildiz, I.F., M. Pierobon, S. Balasubramaniam, and Y. Koucheryavy, "The internet of Bio-Nano things", IEEE Communications Magazine, 53(3), 2015, pp. 32-40.

[4] Ali, N.A. and M. Abu-Elkheir, "Internet of nano-things healthcare applications: Requirements, opportunities, and challenges", in Proceedings of the International Conference on Wireless and Mobile Computing, Networking and Communications (WiMob). 2015.

[5] Al-Rawahi, M.N., T. Sharma, and P. Palanisamy, "Internet of nanothings: Challenges \& opportunities", in Proceedings of the Majan International Conference (MIC). 2018.

[6] Al-Sarawi, S., M. Anbar, K. Alieyan, and M. Alzubaidi, "Internet of Things (IoT) communication protocols: Review", in Proceedings of the International Conference on Information Technology (ICIT). 2017.

[7] Arnall, A.H., Future technologies, today's choices: Nanotechnology, artificial intelligence and robotics; a technical, political and institutional map of emerging technologies, Greenpeace Environmental Trust, London, 2003.

[8] Baker, S.B., W. Xiang, and I. Atkinson, "Internet of Things for Smart Healthcare: Technologies, Challenges, and Opportunities", IEEE Access, 5, 2017, pp. 2652126544.

[9] Bakhshi, T. and S. Shahid, eds., Securing internet of bio-nano things: Ml-enabled parameter profiling of bio- cyber interfaces, Institute of Electrical and Electronics Engineers Inc, 2019.

[10] Balasubramaniam, S. and J. Kangasharju, "Realizing the Internet of Nano Things: Challenges, Solutions, and Applications", Computer, 46(2), 2013, pp. 62-68.

[11] Carvalho Silva, J. de, J. Rodrigues, A. Alberti, P. Solic, and A. Aquino, "LoRaWAN - A low power WAN protocol for Internet of Things: A review and opportunities", in Proceedings of the 2nd International Multidisciplinary Conference on Computer and Energy Science (SpliTech). 2017.

[12] Chude-Okonkwo, U.A.K., R. Malekian, and B.T. Maharaj, "Biologically Inspired Bio-Cyber Interface Architecture and Model for Internet of Bio-NanoThings Applications", Ieee Transactions on Communications, 64(8), 2016, pp. 3444-3455.

[13] Chude-Okonkwo, U.A.K., R. Malekian, B.T. Maharaj, and C.C. Chude, "Bio-Inspired Approach for Eliminating Redundant Nanodevices in Internet of Bio-Nano Things", in Proceedings of the IEEE Globecom Workshops (GC Wkshps). 2015.

[14] Chude-Okonkwo, U.A.K., R. Malekian, B.T. Maharaj, and A.V. Vasilakos, "Molecular Communication and Nanonetwork for Targeted Drug Delivery: A Survey", IEEE Communications Surveys \& Tutorials, 19(4), 2017, pp. 3046-3096.

[15] Dahlqvist, F., M. Patel, A. Rajko, and J. Shulman, Growing opportunities in the Internet of Things https://mck.co/32rvTYi, accessed 09-28-2020.

[16] Dressler, F. and S. Fischer, "Connecting in-body nano communication with body area networks: Challenges and opportunities of the Internet of Nano Things", 18787789, 6(2), 2015, pp. 29-38.

[17] El-Fatyany, A., H. Wang, and S.M. Abd El-atty, "On mixing reservoir targeted drug delivery Modeling-based Internet of Bio-NanoThings", Wireless Networks, 26(5), 2020, pp. 3701-3713.

[18] El-Fatyany, A., H. Wang, S.M. Abd El-atty, and M. Khan, "Biocyber Interface-Based Privacy for Internet of Bio-nano Things", Wireless Pers Commun, 2020.

[19] Feynman, R.P., "There's Plenty of Room at the Bottom", Engineering and Science, 23(5), 1960, pp. 22-36.

[20] Fouad, H., M. Hashem, and A.E. Youssef, "A Nanobiosensors model with optimized bio-cyber communication system based on Internet of Bio-Nano Things for thrombosis prediction", Journal of Nanoparticle Research, 22(7), 2020.

[21] Gul, H., S. Saeed, F.Z.A. Khan, and S. Manzoor, "Potential of Nanotechnology in Agriculture and Crop Protection: A Review", 2014.

[22] Han, B., V.K. Tomer, T.A. Nguyen, A. Farmani, and P. Kumar Singh, eds., Nanosensors for Smart Cities : Micro and Nano Technologies, Elsevier, 2020. 
[23] Hulla, J.E., S.C. Sahu, and A.W. Hayes, "Nanotechnology: History and future", Human \& experimental toxicology, 34(12), 2015, pp. 1318-1321.

[24] Institute of Electrical and Electronics Engineers, "802.15.6-2012 - IEEE Standard for Local and metropolitan area networks: Part 15.6: Wireless Body Area Network"(IEEE 802.15.6).

[25] Jornet, J.M. and I.F. Akyildiz, "Fundamentals of Electromagnetic Nanonetworks in the Terahertz Band", Foundations and Trends ${ }^{\circledR}$ in Networking, 7(2-3), 2012, pp. 77-233.

[26] Jornet, J.M. and I.F. Akyildiz, "Joint Energy Harvesting and Communication Analysis for Perpetual Wireless Nanosensor Networks in the Terahertz Band", IEEE Transactions on Nanotechnology, 11(3), 2012, pp. 570-580.

[27] Kethineni, P., "Applications of internet of nano things: A survey", in Proceedings of the 2nd International Conference for Convergence in Technology (I2CT). 2017.

[28] Khan, T., M. Civas, O. Cetinkaya, N.A. Abbasi, and O.B. Akan, "Nanosensor networks for smart health care", in Nanosensors for Smart Cities : Micro and Nano Technologies, B. Han, V.K. Tomer, T.A. Nguyen, A. Farmani, and P. Kumar Singh, Editors. 2020. Elsevier.

[29] Kirichek, R., R. Pirmagomedov, R. Glushakov, and A. Koucheryavy, "Live substance in cyberspace - Biodriver system", in Proceedings of the 18th International Conference on Advanced Communication Technology (ICACT), Pyeongchang Kwangwoon Do, South Korea. 2016. IEEE.

[30] Lee, J.-S., Y.-W. Su, and C.-C. Shen, "A Comparative Study of Wireless Protocols: Bluetooth, UWB, ZigBee, and Wi-Fi", in Proceedings of the 33rd Annual Conference of the IEEE Industrial Electronics Society, Taipei, Taiwan. 2007.

[31] Lee, S.J., C. Jung, K. Choi, and S. Kim, "Design of wireless nanosensor networks for intrabody application", 15501329, 2015.

[32] National Nanotechnology Initiative, What It Is and How It Works https://www.nano.gov/nanotech-101/what, accessed 09-28-2020.

[33] Nieto-Chaupis, H., "Nanodevices and the Internet of Bio-Nano Things for Modifying Insulin Densities in Pancreatic Beta-Cells Through Electrodynamics of Ca 2+", in Proceedings of the 13th Nanotechnology Materials and Devices Conference (NMDC). 2018.

[34] Nieto-Chaupis, H., "Prospective Usage of the Internet of Bio-Nano Things For The Very Anticipated Detection of Charged Giants Proteins in Type-2 Diabetes Patients: Stopping the Progress of Diabetic Nephropathy with Nanomedicine", in Proceedings of the 16th LACCEI International Multi-Conference for Engineering, Education, and Technology: "Innovation in Education and Inclusion". 2018. Latin American and Caribbean Consortium of Engineering Institutions.
[35] Paukstadt, U., G. Strobel, and S. Eicker, "Understanding Services in the Era of the Internet of Things: A Smart Service Taxonomy", in Proceedings of the 27th European Conference on Information Systems (ECIS). 2019: Stockholm, Sweden.

[36] Piro, G., G. Boggia, and L.A. Grieco, "On the design of an energy-harvesting protocol stack for Body Area Nano-NETworks", Nano Communication Networks, 6(2), 2015, pp. 74-84.

[37] Pramanik, P.K.D., A. Solanki, A. Debnath, A. Nayyar, S. El-Sappagh, and K.-S. Kwak, "Advancing Modern Healthcare With Nanotechnology, Nanobiosensors, and Internet of Nano Things: Taxonomies, Applications, Architecture, and Challenges", IEEE Access, 8, 2020, pp. 65230-65266.

[38] Qadri, Y.A., A. Nauman, Y. Bin Zikria, A.V. Vasilakos, and S.W. Kim, "The Future of Healthcare Internet of Things: A Survey of Emerging Technologies", IEEE Communications Surveys and Tutorials, 22(2), 2020, pp. 1121-1167.

[39] Roco, M.C., "Nanoparticles and Nanotechnology Research", Journal of Nanoparticle Research, 1(1), 1999, pp. 1-6.

[40] Scheer, A.-W., ARIS - Business Process Modeling, Springer Berlin Heidelberg, Berlin, Heidelberg., 2000.

[41] Sicari, S., A. Rizzardi, G. Piro, A. Coen-Porisini, and L.A. Grieco, "Beyond the smart things: Towards the definition and the performance assessment of a secure architecture for the Internet of Nano-Things", Computer Networks, 162, 2019, p. 106856.

[42] Silvestre, C., D. Duraccio, and S. Cimmino, "Food packaging based on polymer nanomaterials", Progress in Polymer Science, 36(12), 2011, pp. 1766-1782.

[43] Stelzner, M., F. Dressler, and S. Fischer, "Function Centric Nano-Networking: Addressing nano machines in a medical application scenario", 18787789, 14, 2017, pp. 2939.

[44] Stix, G., "Little big science. Nanotechnology", Scientific American, 285(3), 2001, pp. 32-37.

[45] Tamás, B. and Z. Gál, Overview of the Internet of Things Short Range Communication Technologies, 2015.

[46] Vom Brocke, J., A. Simons, B. Niehaves, K. Riemer, R. Plattfaut, and A. Cleven, "Reconstructing the giant: On the importance of rigour in documenting the literature search process", in Proceedings of the 17th European Conference on Information Systems, ECIS 2009, Verona, Italy. 2009.

[47] Webster, J. and R.T. Watson, "Analyzing the past to prepare for the future: Writing a literature review", MIS Quarterly, 2002, pp. xiii-xxiii.

[48] Zeng, W., Y. Zhao, K. Ou, and W. Song, "Research on cloud storage architecture and key technologies", in Proceedings of the 2nd International Conference on Interaction Sciences Information Technology, Culture and 
Human - ICIS '09, S. Sohn, L. Chen, S. Hwang, K. Cho, S.

Kawata, K. Um, F.I.S. Ko, K.D. Kwack, J.H. Lee, G. Kou,

K. Nakamura, A.C.M. Fong, and P. Ma, Editors, Seoul,

Korea. 2009. 